\title{
Comparison of vibrational and electronic contributions to van der Waals interactions
}

\author{
Kyungwha Park, $, 1,2, *, \dagger$ Mark R. Pederson, ${ }^{1, \ddagger}$ and Amy Y. Liu ${ }^{2, \S}$ \\ ${ }^{1}$ Center for Computational Materials Science, Code 6392, Naval Research Laboratory, Washington, D.C. 20375, USA \\ ${ }^{2}$ Department of Physics, Georgetown University, Washington, D.C. 20057, USA \\ (Received 13 July 2005; revised manuscript received 29 March 2006; published 22 May 2006)
}

\begin{abstract}
The van der Waals interaction can be caused by either ionic vibrations or instantaneous electronic motion relative to the atomic center. In this study, the vibrational contribution to the van der Waals interaction is formulated by considering the interaction between induced dipoles caused by the infrared-active normal modes of a neutral molecule. Using the derived formula, the contribution is quantified, within the density-functional theory formalism, using a screened, i.e., self-consistent, vibrational polarizability. Applications for several neutral nonpolar dimers are presented. It is found that the vibrational contributions for the dimers are substantially smaller than their electronic contributions. The ratio of the vibrational to electronic contributions depends strongly on the ratio of the screened vibrational to electronic polarizabilities and on the ratio of the frequency of the strongest infrared-active mode to an ionization energy.
\end{abstract}

DOI: 10.1103/PhysRevB.73.205116 PACS number(s): 31.15.Ew, 34.20.-b, 33.20.Ea, 33.15.Kr

\section{INTRODUCTION}

Chemical and biological molecules ranging from rare-gas diatomic molecules to proteins, DNA, RNA, and fullerenes $\left(\mathrm{C}_{60}\right)$ are held together by noncovalent weak interactions such as van der Waals (vdW) interactions and/or hydrogen bonds. ${ }^{1-8}$ The vdW attraction between fullerene molecules plays a crucial role in characterizing their physical, chemical, and mechanical properties. ${ }^{3}$ The vdW forces acting on proteins are partly responsible for their structure and flexibility. ${ }^{4}$ The strength of the vdW interaction for small molecules is typically on the order of 5-20 meV. Quantum mechanically, electrons localized around nuclei can move instantaneously, which causes a fluctuating dipole moment within an atom. This induces dipole moments in neighboring atoms. The interaction between the fluctuating dipole moment and the induced dipole moments is attractive and is called the dispersion or vdW interaction. For a collection of neutral atoms and molecules that do not have permanent dipole moments, the vdW interaction plays a major role in binding.

As a simple model for the vdW interaction, Drude described an atom in terms of positive and negative charges, representing the nucleus and electrons, that are joined by a spring. From this ansatz it follows that the total zero-point energy of two neutral atoms is decreased by a term that scales as $1 / R^{6} .^{9-13}$ This Drude model has been modified and generalized to understand intermolecular interactions between small molecules such as hydrogen molecules, rare-gas dimers, and alkali dimers at long distances. ${ }^{14-16}$ In addition, the vdW interactions for the small molecules have been extensively studied by improving calculations of dynamic and static polarizabilities using coupled multiconfiguration ${ }^{17}$ or configuration interaction methods. ${ }^{18,19}$ Additional strategies have been to theoretically determine upper and lower bounds of the vdW coefficients based on the Padé approximation and sum rules applied to the calculations of the polarizabilities. $^{20-25}$

Density-functional theory (DFT) has been greatly successful in describing many physical and chemical properties in various many-electron systems. Rapcewicz and $\mathrm{Ashcroft}^{26}$ have formulated the fluctuations in the electronic charge density as response or correlation density functions and obtained the attractive interaction in the asymptotic limit using an effective density $\rho_{\text {eff }}=\sqrt{\rho\left(\vec{r}_{1}\right) \rho\left(\vec{r}_{2}\right)}$, where $\rho\left(\vec{r}_{1}\right)$ and $\rho\left(\vec{r}_{2}\right)$ are the densities of electrons at positions $\vec{r}_{1}$ and $\vec{r}_{2}$ in two separate atoms. Their calculated $\mathrm{vdW}$ coefficients for rare-gas atoms were in good agreement with experiment. Starting with the work of Rapcewicz and Ashcroft, ${ }^{26}$ Andersson et al. ${ }^{27}$ have developed the approximations to the $\mathrm{vdW}$ interactions for dimers of various atoms. In this work, dynamic atomic polarizabilities were determined using the DFT-calculated ground-state charge densities and a spatial cutoff in the response frequency. Dobson and collaborators ${ }^{28}$ have derived a $\mathrm{vdW}$ interaction from density-density response functions that satisfy charge conservation and reciprocity. Van Gisbergen et $a .^{29}$ and Misquitta et al. ${ }^{30}$ have respectively used time dependent DFT and symmetry-adapted perturbation theory with Kohn-Sham orbitals to calculate the vdW interactions for polyatomic molecules from dynamic atomic polarizabilities.

Fluctuations in electronic charge density can also be induced by vibrations of atoms within a molecule. To distinguish this attractive term from the effect due to instantaneous electronic motion, this is hereafter referred to as a vibrational contribution to the vdW interaction. The vibrational vdW interaction can be calculated using DFT because it only requires the computation of vibrational normal modes and the corresponding frequencies for each monomer participating in the vdW bonding. It has been known that the electronic dispersion energy scales with the square of an atomic polarizability, ${ }^{10-13}$ which leads to the fact that the binding energy increases quadratically with increasing molecular weight. For some molecules, vibrational polarizabilities have been experimentally determined to be on the same order as their electronic polarizabilities. $^{31}$ This observation motivated us to investigate the vibrational $\mathrm{vdW}$ interaction as a function 
of molecular weight and to compare it to the electronic contribution.

In this paper, the vibrational $\mathrm{vdW}$ interaction is formulated using an analogy of the Drude model..$^{9-13,16}$ The vibrational $\mathrm{vdW}$ is quantified for nine different nonpolar dimers using an all-electron Gaussian-orbital-based DFT code, NRLMOL (Naval Research Laboratory Molecular Orbital Library), ${ }^{32}$ within the generalized gradient approximation (GGA). ${ }^{33}$ Then, to lowest order, the electronic contributions are estimated using (i) Kohn-Sham orbitals and orbital energies and (ii) approximate London's formula. ${ }^{10-13}$ The calculated vibrational contribution (using a screened vibrational polarizability) is compared to the empirical electronic one for the examined dimers. The dependence of the $\mathrm{vdW}$ interactions on atomic number is discussed and an upper limit for the ratio of the vibrational to electronic contributions is offered.

\section{FORMALISM}

\section{A. Drude model}

In the one-dimensional Drude model, ${ }^{9-13,16}$ an atom with a fluctuating dipole moment is described by a linear harmonic oscillator in which positive and negative charges are joined by a spring with force constant $k$. We consider two identical neutral atoms with a separation, $R$, that is much larger than the separations between charges, $\left|x_{1}\right|$ and $\left|x_{2}\right|$, on the two atoms. Under these conditions, the lowest-order Coulomb interaction between the two atoms (oscillators) becomes a dipole-dipole interaction. Thus, the total Hamiltonian of the system is written as

$$
\mathcal{H}_{\mathrm{e}}=\frac{m \dot{x}_{1}^{2}}{2}+\frac{k x_{1}^{2}}{2}+\frac{m \dot{x}_{2}^{2}}{2}+\frac{k x_{2}^{2}}{2}-\frac{2 e^{2} x_{1} x_{2}}{R^{3}} .
$$

If the coupled oscillators are decoupled using a normal-mode transformation, the zero-point energy of the system is reduced from that of the sum of the two uncoupled oscillators, $\hbar \omega_{0}$, where $\omega_{0}=\sqrt{k / m}$, by

$$
\Delta U_{e}=-\hbar \omega_{0} \frac{1}{8}\left(\frac{2 e^{2}}{k R^{3}}\right)^{2} .
$$

This energy difference, which is inversely proportional to the sixth power of the separation of the two oscillators, is the $\mathrm{vdW}$ interaction energy.

\section{B. Vibrational contribution}

While the Drude model was originally used to qualitatively explain the electronic $\mathrm{vdW}$ interaction, it also points to the fact that other mechanisms such as infrared vibrational modes can yield a vdW interaction as well. To calculate the vibrational vdW interaction energy, we generalize the onedimensional Drude model to a system of many threedimensional harmonic oscillators. Suppose that a system consists of $N_{m}$ molecules, each of which comprises $N$ atoms. The $N$ three-dimensional coupled harmonic oscillators corresponding to individual atoms in a molecule can be transformed to $3 \mathrm{~N}$ one-dimensional uncoupled harmonic oscilla- tors in normal-mode coordinates. Among the $3 N$ normal modes, only $N_{\text {IR }}$ infrared (IR) active modes contribute to the vibrational vdW interaction. Thus, similar to Eq. (1), the total Hamiltonian, in terms of the normal-mode coordinates $Q_{i v}$ and momenta $P_{i \nu}$, becomes

$$
\begin{gathered}
\mathcal{H}_{v}=\sum_{\nu=1}^{N_{m}} \sum_{i=1}^{N_{\mathrm{IR}}}\left(\frac{P_{i \nu}^{2}}{2 m_{i \nu}}+\frac{1}{2} m_{i \nu} \omega_{i \nu}^{2} Q_{i \nu}^{2}\right)+\sum_{\nu>\nu^{\prime}}^{N_{m}} \sum_{\nu^{\prime}=1}^{N_{m}} V_{d}\left(\nu, \nu^{\prime}\right), \\
V_{d}\left(\nu, \nu^{\prime}\right)=\sum_{i j} \frac{\vec{\mu}_{i \nu} \cdot \vec{\mu}_{j \nu^{\prime}}-3\left(\vec{\mu}_{i \nu} \cdot \hat{R}_{\nu \nu^{\prime}}\right)\left(\vec{\mu}_{j \nu^{\prime}} \cdot \hat{R}_{\nu \nu^{\prime}}\right)}{R_{\nu \nu^{\prime}}^{3}},
\end{gathered}
$$

where $V_{d}$ is the dipole-dipole interaction, $\omega_{i \nu}$ is the frequency of the $i$ th IR-active normal mode for the $\nu$ th molecule with the effective mass of $m_{i v}, \vec{\mu}_{i \nu}$ is a dynamic dipole moment induced by a displacement along the corresponding normalmode direction, and $R_{\nu \nu^{\prime}}$ is a separation between a pair of molecules $\left(\nu, \nu^{\prime}\right)$. Here the summations of the last term in Eq. (3) run over all pairs of the molecules and the summation in Eq. (4) runs over all IR active modes. The dynamic dipole moment is expanded according to

$$
\vec{\mu}_{i \nu}\left(Q_{i \nu}\right)=\vec{\mu}_{i \nu}\left(Q_{i \nu}=0\right)+Q_{i \nu} \frac{\partial \vec{\mu}}{\partial Q_{i \nu}}+\cdots,
$$

where $\vec{\mu}_{i \nu}\left(Q_{i \nu}=0\right)=0$ because the molecules considered do not carry permanent dipole moments. The $x$ component of the dynamic dipole moment of the first molecule can be rewritten as

$$
\mu_{i 1}^{x}=Q_{i 1} \frac{\partial \mu_{x}}{\partial Q_{i 1}}=Q_{i 1} \sum_{k=1}^{3 N} \frac{\partial \mu_{x}}{\partial R_{k}} \frac{\partial R_{k}}{\partial Q_{i 1}}=Q_{i 1} \sum_{k=1}^{3 N} \frac{\partial F_{k}}{\partial E_{x}} \frac{\partial R_{k}}{\partial Q_{i 1}} \equiv Q_{i 1} A_{i}^{x},
$$

where the sum is over atoms and Cartesian coordinates, $R_{k}$ is an atomic coordinate, $F_{k}$ is a component of the force on an atom, $E_{x}$ is the $x$ component of an applied electric field, $A_{i}^{x}$ represents an effective charge, and $\partial R_{k} / \partial Q_{i 1}$ is the $k$ th element of the $i$ th eigenvector (normal mode). The induced dipole-dipole interaction between the $\nu$ th and $\nu^{\prime}$ th molecules can be written, in terms of the normal-mode coordinates and effective charges, as

$$
\begin{aligned}
V_{d}\left(\nu, \nu^{\prime}\right)= & \sum_{i j} Q_{i \nu} Q_{j \nu^{\prime}}\left[A _ { i } ^ { x } A _ { j } ^ { x } \left(\cos \theta_{1} \cos \theta_{2} \cos \phi\right.\right. \\
& \left.-2 \sin \theta_{1} \sin \theta_{2}\right)+A_{i}^{y} A_{j}^{y} \cos \phi \\
& +A_{i}^{z} A_{j}^{z}\left(\sin \theta_{1} \sin \theta_{2} \cos \phi-2 \cos \theta_{1} \cos \theta_{2}\right) \\
& +A_{i}^{x} A_{j}^{y} \cos \theta_{1} \sin \phi+A_{i}^{y} A_{j}^{x} \cos \theta_{2} \sin \phi \\
& +A_{i}^{x} A_{j}^{z}\left(\cos \theta_{1} \sin \theta_{2} \cos \phi+2 \sin \theta_{1} \cos \theta_{2}\right) \\
& +A_{i}^{z} A_{j}^{x}\left(\sin \theta_{1} \cos \theta_{2} \cos \phi+2 \cos \theta_{1} \sin \theta_{2}\right) \\
& \left.+A_{i}^{y} A_{j}^{z} \sin \theta_{2} \sin \phi-A_{i}^{z} A_{j}^{y} \sin \theta_{1} \sin \phi\right] / R_{\nu \nu^{\prime}}^{3} \\
\equiv & \sum_{i j} \frac{Q_{i \nu} Q_{j \nu^{\prime}} K_{i j}\left(\theta_{1}, \theta_{2}, \phi\right)}{R_{\nu \nu^{\prime}}^{3}}
\end{aligned}
$$


where the summation on $i(j)$ runs over all IR modes for the $\nu$ th $\left(\nu^{\prime}\right.$ th) molecule. Here we used the coordinate systems following van der Merwe. ${ }^{34} \theta_{1}$ and $\theta_{2}$ are the angles between the separation vector $\vec{R}_{\nu \nu^{\prime}}$ and the $z$ axes of molecule $\nu$ and $\nu^{\prime}$. $x_{\nu}\left(x_{\nu^{\prime}}\right)$ is coplanar with $\vec{R}_{\nu \nu^{\prime}}$ and $z_{\nu}\left(z_{\nu^{\prime}}\right)$. $\phi$ is the angle between the projected vectors of $z_{\nu}$ and $z_{\nu^{\prime}}$ onto a plane perpendicular to $\vec{R}_{\nu \nu^{\prime}} . K_{i j}\left(\theta_{1}, \theta_{2}, \phi\right)$ has units of the square of charge. As shown in Eq. (7), the vibrational vdW interaction can be anisotropic. For example, the vibrational $\mathrm{vdW}$ interactions for two NCCN molecules and two benzene molecules are highly anisotropic. To obtain an analytical expression for the vibrational contribution, we consider $V_{d}$ as a small perturbation to the unperturbed system [uncoupled harmonic oscillators, Eq. (3) without $V_{d}$ ] in which the normalized ground-state eigenvector is $\Psi_{0} \equiv\left|0_{\nu}, 0_{\nu^{\prime}}\right\rangle$. The first-order term in $V_{d}$ vanishes because $\left\langle\Psi_{0}\left|V_{d}\right| \Psi_{0}\right\rangle$ $=\left\langle 0_{\nu}\left|Q_{i \nu}\right| 0_{\nu}\right\rangle\left\langle 0_{\nu^{\prime}}\left|Q_{j \nu^{\prime}}\right| 0_{\nu^{\prime}}\right\rangle=0$. Thus, the zero-point energy difference is, to lowest order,

$$
\begin{aligned}
\Delta U_{v} & =\sum_{k} \frac{\left|\left\langle\Psi_{0}\left|V_{d}\right| \Psi_{k}\right\rangle\right|^{2}}{\epsilon_{0}-\epsilon_{k}}=-\sum_{i j} \frac{\left|\left\langle 0\left|Q_{i}\right| 1\right\rangle\left\langle 0\left|Q_{j}\right| 1\right\rangle K_{i j}\right|^{2}}{R^{6} \hbar\left(\omega_{i}+\omega_{j}\right)} \\
& =-\sum_{i j} \frac{K_{i j}^{2} \hbar}{4\left(\omega_{i}+\omega_{j}\right) m_{i} \omega_{i} m_{j} \omega_{j} R^{6}} \equiv-\frac{C_{6}^{v}}{R^{6}},
\end{aligned}
$$

where $\epsilon_{0}$ is the zero-point energy of the unperturbed system, $\epsilon_{k}$ is an excited energy of the unperturbed system, $|1\rangle$ is the first-excited state of one molecule, and $\omega_{i}$ is the angular frequency of the $i$ th normal mode with an effective mass of $m_{i}$. $\left\langle 0\left|Q_{i}\right| 1\right\rangle=\sqrt{\hbar} / \sqrt{2 m_{i} \omega_{i}}$ is derived using the ground and excited states of harmonic oscillators in standard quantum mechanics. $\Delta U_{v}$ is proportional to the square of the IR absorption intensity.

\section{Electronic contribution}

The electronic contribution is calculated using two methods: (i) Kohn-Sham orbitals and orbital energies and (ii) the approximate London's formula. ${ }^{13,35}$ Using second-order perturbation theory, the electronic $\mathrm{vdW}$ interaction is written as

$$
\Delta U_{\mathrm{e}}^{(2)}=\sum_{\alpha \neq 0, \beta \neq 0} \frac{\left|\left\langle\Phi_{0}^{A} \Phi_{0}^{B}\left|V_{A B}\right| \Phi_{\alpha}^{A} \Phi_{\alpha}^{B}\right\rangle\right|^{2}}{\mathcal{E}_{0}^{A}+\mathcal{E}_{0}^{B}-\mathcal{E}_{\alpha}^{A}-\mathcal{E}_{\beta}^{B}}
$$

where $V_{A B}$ is the Coulomb interaction between electrons within two molecules, $\mathcal{E}_{0}^{A}$ and $\mathcal{E}_{0}^{B}$ are eigenvalues of the ground states $\Phi_{0}^{A}$ and $\Phi_{0}^{B}$ for molecule $A$ and molecule $B$, and $\mathcal{E}_{\alpha}^{A}$ and $\mathcal{E}_{\beta}^{B}$ are eigenvalues of their excited states $\Phi_{\alpha}^{A}$ and $\Phi_{\beta}^{B}$. If we consider only the lowest-order term of $V_{A B}$, which is a dipole-dipole interaction, then Eq. (10) is approximated to

$$
\begin{aligned}
\Delta U_{e}^{(2)} & =\frac{4}{R^{6}} \sum_{i=1}^{n_{o} / 2} \sum_{j=\left(n_{o} / 2\right)+1}^{\infty} \sum_{k=1}^{n_{o} / 2} \sum_{l=\left(n_{o} / 2\right)+1}^{\infty} \frac{\left[\left\langle\phi_{i}^{A}|\vec{r}| \phi_{j}^{A}\right\rangle\left\langle\phi_{k}^{B}|\vec{r}| \phi_{l}^{B}\right\rangle-3\left\langle\phi_{i}^{A}|\vec{r} \cdot \hat{R}| \phi_{j}^{A}\right\rangle\left\langle\phi_{k}^{B}|\vec{r} \cdot \hat{R}| \phi_{l}^{B}\right\rangle\right]^{2}}{\epsilon_{i}^{A}-\epsilon_{j}^{A}+\epsilon_{k}^{B}-\epsilon_{l}^{B}}, \\
& \equiv-\frac{C_{6}^{C}[\mathrm{cal}]}{R^{6}},
\end{aligned}
$$

using occupied (unoccupied) Kohn-Sham orbitals $\phi_{i}^{A}$ and $\phi_{k}^{B}\left(\phi_{j}^{A}\right.$ and $\left.\phi_{l}^{B}\right)$ and orbital energies $\epsilon_{i}^{A}$ and $\epsilon_{k}^{B}\left(\epsilon_{j}^{A}\right.$ and $\epsilon_{l}^{B}$ ). Here $n_{0}$ is the total number of electrons in a molecule and $\hat{R}$ is a unit vector along the separation between the two molecules considered. Kohn-Sham orbitals and orbital energies are obtained using all-electron Gaussian basis sets within the GGA. A factor of 4 arises from spin indices. The expression for $C_{6}^{e}[\mathrm{cal}]$ is similar to that used in Refs. 36 and 37. Before continuing, we review how Eq. (11) can be simplified to the approximate London's formula. ${ }^{13,35}$ The $x x$ component of a static atomic polarizability tensor neglecting screening, hereafter referred to as the bare polarizability tensor, is defined as

$$
\alpha_{e, x x}^{\mathrm{bare}}=2 \sum_{\alpha \neq 0} \frac{\left|\left\langle\Phi_{0}|\hat{x} \cdot \vec{r}| \Phi_{\alpha}\right\rangle\right|^{2}}{\mathcal{E}_{\alpha}-\mathcal{E}_{0}}=4 \sum_{i=1}^{n_{0} / 2} \sum_{j=n_{0} / 2+1}^{\infty} \frac{\left|\left\langle\phi_{i}|\hat{x} \cdot \vec{r}| \phi_{j}\right\rangle\right|^{2}}{\epsilon_{j}-\epsilon_{i}} .
$$

Assume that there is only a single dominant dipole matrix element, that the $\mathrm{vdW}$ interaction is isotropic, and that the denominator in Eq. (13) is dominated by an ionization energy $I$. Then the electronic contribution, Eq. (11), becomes

$$
\Delta U_{e}^{(2)} \approx-\frac{3}{4 R^{6}}\left(\alpha_{e}^{\text {bare }}\right)^{2} I .
$$

If a bare polarizability $\alpha_{e}^{\text {bare }}$ in Eq. (14) is replaced by a screened polarizability $\alpha_{e}^{\text {scr }}$, then this becomes identical to the approximate London's formula. 
TABLE I. The atomic number $Z$, calculated static vibrational polarizability $\alpha_{v}^{\text {cal }}$, and bare and screened electronic polarizability $\alpha_{e, \text { bare }}^{\text {cal }}$ and $\alpha_{e, \text { scr }}^{\text {cal }}$ are presented along with values ${ }^{31}$ obtained from infrared absorption and dielectric measurements $\left(\alpha_{v}^{\exp }\right)$ and from optical measurements $\left(\alpha_{e}^{\exp }\right)$, respectively, in units of $\AA^{3}$. Here $\omega_{h}$ indicates the wave number of the strongest IR active normal mode (could be degenerate) and $I$ denotes a calculated ionization energy. Notice that $1 \mathrm{~cm}^{-1}=1.2375 \times 10^{-4} \mathrm{eV}$.

\begin{tabular}{lcccccccc}
\hline \hline & $Z$ & $\alpha_{v}^{\text {cal }}$ & $\alpha_{v}^{\text {exp }}$ & $\alpha_{e, \text { bare }}^{\text {cal }}$ & $\alpha_{e, \text { scr }}^{\text {cal }}$ & $\alpha_{e}^{\text {exp }}$ & $\omega_{h}(1 / \mathrm{cm})$ & $I(\mathrm{eV})$ \\
\hline $\mathrm{CH}_{4}$ & 10 & 0.039 & $0.03,0.03$ & 3.59 & 2.63 & 2.60 & 3071 & 14.04 \\
$\mathrm{NCCN}$ & 26 & 0.94 & $0.91,3.30$ & 11.62 & 5.15 & 4.71 & 233 & 13.09 \\
$\mathrm{C}_{6} \mathrm{H}_{6}$ & 42 & 0.35 & $0.29,0.75$ & 19.94 & 10.5 & 9.96 & 659 & 9.27 \\
$\mathrm{SiF}_{4}$ & 50 & 2.07 & $1.75,2.14$ & 5.08 & 3.73 & 3.32 & 969 & 14.4 \\
$\mathrm{SF}_{6}$ & 70 & 2.30 & $2.29,2.06$ & 8.18 & 5.15 & 4.49 & 846 & 13.77 \\
$\mathrm{CCl}_{4}$ & 74 & 1.41 & $0.84,0.95$ & 20.26 & 11.0 & 10.24 & 711 & 10.56 \\
$\mathrm{SnCl}_{4}$ & 118 & 4.35 & $6.36,4.09$ & 26.6 & 14.7 & 13.97 & 380 & 10.84 \\
$\mathrm{SnBr}_{4}$ & 190 & 3.98 & $3.23,3.49$ & 38.4 & 20.2 & 18.57 & 266 & 9.92 \\
$\mathrm{SnI}_{4}$ & 262 & 3.94 & none, 5.75 & 73.96 & 30.3 & 26.19 & 208 & 8.91 \\
\hline \hline
\end{tabular}

\section{RESULTS AND COMPARISON OF VIBRATIONAL TO ELECTRONIC CONTRIBUTIONS}

We now present the calculation of coefficients of the vibrational contribution $C_{6}^{v}$ for nine different nonpolar dimers: $\mathrm{CH}_{4}-\mathrm{CH}_{4}, \mathrm{NCCN}-\mathrm{NCCN}, \mathrm{C}_{6} \mathrm{H}_{6}-\mathrm{C}_{6} \mathrm{H}_{6}, \mathrm{SiF}_{4}-\mathrm{SiF}_{4}$, $\mathrm{SF}_{6}-\mathrm{SF}_{6}, \mathrm{CCl}_{4}-\mathrm{CCl}_{4}, \mathrm{SnCl}_{4}-\mathrm{SnCl}_{4}, \mathrm{SnBr}_{4}-\mathrm{SnBr}_{4}$, and $\mathrm{SnI}_{4}$ $-\mathrm{SnI}_{4}$. The geometry of each monomer is optimized using all-electron Gaussian-orbital-based NRLMOL within the GGA. Then all atoms in a monomer are displaced from the equilibrium positions along the $\pm x, \pm y$, and $\pm z$ axes, which leads to $6 N$ displaced geometries for systems without any symmetry. The energies of the displaced geometries are calculated self-consistently using NRLMOL. For small displacements, one can calculate, within the harmonic-oscillator approximation, normal modes and their corresponding frequencies and effective masses by solving eigenvalue problems. ${ }^{38}$ The IR absorption intensity of the $i$ th normal mode is, to first order, proportional to $\left|d \vec{\mu} / d Q_{i}\right|^{2}$, where $\vec{\mu}$ is an electric dipole moment. An external electric field is applied to the optimized geometry along various directions to compute the IR intensity using the relationship $\partial \mu_{i} / \partial R_{k}$ $=\partial F_{k} / \partial E_{i}$. The energies and forces of the geometries in the presence of an external electric field are calculated selfconsistently. We determine IR active modes as well as the dynamic dipole moments [effective charges $A_{i}^{x, y, z}$ or $\left.K_{i j}\left(\theta_{1}, \theta_{2}, \phi\right)\right]$ for each IR mode. The vibrational polarizability tensor is calculated as a sum of the square of all effective charges weighted by vibrational frequencies of all normal modes, ${ }^{40}$

$$
\alpha_{v, \mu \nu}=\sum_{i} A_{i}^{\mu} \omega_{i}^{-2}\left(A_{i}^{\nu}\right)^{T}
$$

where the summation runs over all normal modes with frequency $\omega_{i}$, and $\mu, \nu=x, y$, and $z$. This is a screened vibrational polarizability tensor. In most cases, the calculated mean values of the polarizability are in good agreement with the two sets of experimental values obtained from infrared absorption and dielectric measurements ${ }^{31}$ (Table I). The values of $C_{6}^{v}$ calculated for the nine dimers using Eq. (9) are shown in Table II. The vdW interactions are isotropic for all of the molecules examined except for linear NCCN and planar $\mathrm{C}_{6} \mathrm{H}_{6}$ molecules in which the interactions are calculated at $\theta_{1}=\theta_{2}=0$ and $\phi=0$. (The reason that the anisotropy of the vdW interaction is not pursued will be provided later in this section.) The value of $C_{6}^{v}$ increases by three orders of magnitude as molecular weight increases by a factor of 30 (Table II and Fig. 1) despite apparent fluctuations.

The calculated value of $C_{6}^{e}[\mathrm{cal}]$ increases substantially with increasing molecular weight (Table II and Fig. 2) as expected. The values of $C_{6}^{e}[\mathrm{cal}]$ for the nine dimers are much greater (by a factor of 2 or 3 ) than the corresponding theoretical $^{39}$ and empirical values, $C_{6}^{e}[\mathrm{emp}]$, calculated from construction of dipole oscillator strength distributions (DOSD) $23,24,41,42$ and from the approximate London's formula [same as Eq. (14) but $\alpha_{e}^{\text {bare }}$ is now replaced by $\alpha_{e}^{\text {scr }}$. This discrepancy is due to the absence of screening of electronic charges in Eq. (11). A screened atomic polarizability tensor $\alpha_{e, \mu \nu}^{\mathrm{scr}}$ is defined as

$$
\alpha_{e, \mu \nu}^{\mathrm{scr}}=-\frac{\partial}{\partial E_{\nu}} \frac{\partial \mathcal{E}}{\partial E_{\mu}},
$$

where $\mathcal{E}$ is the total energy of the system and $E_{\mu}$ is the $\mu$ component of an external electric field. The screened atomic polarizabilities, $\alpha_{e, \text { scr }}^{\text {cal }}$, calculated using Eq. (16) agree well with the experimental values, $\alpha_{e}^{\exp }$ (Ref. 31), as shown in Table I. But the values of $\alpha_{e, s c r}^{\text {cal }}$ are much smaller than those of $\alpha_{e \text {, bare }}^{\mathrm{cal}}$ for the nine dimers. When the same formula, Eq. (11), is applied to rare-gas dimers such as $\mathrm{He}-\mathrm{He}, \mathrm{Ne}-\mathrm{Ne}$, and $\mathrm{Ar}-\mathrm{Ar}$, the values of $C_{6}^{e}[\mathrm{cal}]$ agree with experimental (not shown), empirical (Ref. 25), and other theoretical values (Refs. 27 and 29) for light atoms such as He but not for heavy atoms (Table III). This observation is consistent with the results reported by Refs.36 and 37 even when an exact exchange $(\mathrm{EXX})$ or exchange only (x-only) functional was used. Our calculated $C_{6}^{e}[\mathrm{cal}]$ values for the rare-gas dimers are outside of the theoretical upper and lower bounds obtained from the Padé approximation and the sum rules. ${ }^{20-22}$ 
TABLE II. The atomic number $Z$, calculated values of $C_{6}^{v}$ [using Eq. (9)], empirical values of $C_{6}^{e}$ from Refs. 23, 24, 39, 41, and 42, and Eq. (14) (with $\alpha_{e, \text { scr }}^{\text {cal }}$ ), and calculated values of $C_{6}^{e}$ [using Eq. (12)] in units of (hartree $\cdot a_{B}^{6}$ ), and their ratios, are shown for nine different dimers of the same nuclear species.

\begin{tabular}{|c|c|c|c|c|c|c|c|}
\hline \multirow[b]{2}{*}{$\mathrm{CH}_{4}$} & \multirow{2}{*}{$\begin{array}{l}Z \\
10\end{array}$} & \multirow{2}{*}{$\frac{C_{6}^{v}}{0.00036}$} & \multicolumn{2}{|c|}{$C_{6}^{e}[\mathrm{emp}]$} & \multirow{2}{*}{$\frac{C_{6}^{e}[\mathrm{cal}]}{223.6}$} & \multirow{2}{*}{$\frac{C_{6}^{e}[\mathrm{cal}]\left(\alpha_{e, \mathrm{scr}}^{\mathrm{cal}} / \alpha_{e, \mathrm{bare}}^{\mathrm{cal}}\right)^{2}}{120.0}$} & \multirow{2}{*}{$\frac{C_{6}^{v} / C_{6}^{e}[\mathrm{emp}]^{\mathrm{a}}}{2 \times 10^{-6}}$} \\
\hline & & & $158.4^{\mathrm{b}}$ & $121.9^{\star}$ & & & \\
\hline $\mathrm{NCCN}$ & 26 & 0.0603 & & $435.8^{*}$ & 1204 & 375 & $1 \times 10^{-4}$ \\
\hline $\mathrm{C}_{6} \mathrm{H}_{6}$ & 42 & 0.0043 & $\begin{array}{l}1723^{\mathrm{c}} \\
1163^{\mathrm{d}}\end{array}$ & $1284^{\star}$ & 4354 & 1207.3 & $2 \times 10^{-6}$ \\
\hline $\mathrm{SiF}_{4}$ & 50 & 0.37 & $330.2^{\mathrm{e}}$ & $251.5^{\star}$ & 598.7 & 322.8 & $1 \times 10^{-3}$ \\
\hline $\mathrm{SF}_{6}$ & 70 & 0.67 & $585.8^{\mathrm{f}}$ & $458.5^{\star}$ & 1434 & 568.4 & $1 \times 10^{-3}$ \\
\hline $\mathrm{CCl}_{4}$ & 74 & 0.22 & & $1604^{\star}$ & 5565 & 1640.5 & $1 \times 10^{-4}$ \\
\hline $\mathrm{SnCl}_{4}$ & 118 & 0.54 & & $2941^{\star}$ & 8263 & 2523.5 & $2 \times 10^{-4}$ \\
\hline $\mathrm{SnBr}_{4}$ & 190 & 0.40 & & $5081^{\star}$ & 14873 & 4115.7 & $8 \times 10^{-5}$ \\
\hline $\mathrm{SnI}_{4}$ & 262 & 0.37 & & $10268^{\star}$ & 38383 & 6442.1 & $4 \times 10^{-5}$ \\
\hline
\end{tabular}

${ }^{a}$ The values of $C_{6}^{e}[\mathrm{emp}]$ were taken from the empirical results using dipole oscillator distribution ${ }^{23,24,41,42}$ when they are available. Otherwise, they were estimated from $3 I\left(\alpha_{e, \text { scr }}^{\text {cal }}\right)^{2} / 4$. Those values are marked by $\star$. ${ }^{b}$ Reference 23 .

${ }^{\mathrm{c}}$ Reference 41.

${ }^{\mathrm{d}}$ Reference 39.

eReference 42.

${ }^{\mathrm{f}}$ Reference 24.

This is expected because a bare atomic polarizability was used in our calculation instead of a screened static polarizability as discussed in Sec. II. C. The bare polarizability tends to be greater than the screened polarizability for heavy atoms. This is further supported by the observation that weighting $C_{6}^{e}[\mathrm{cal}]$ by $\left(\alpha_{e, \text { scr }}^{\mathrm{cal}} / \alpha_{e, \text { bare }}^{\mathrm{cal}}\right)^{2}$ yields values closer to $C_{6}^{e}[\mathrm{emp}]$ (Tables I and II).

For a dimer consisting of like monomers, $C_{6}^{v}$ $\approx 0.5 \hbar \omega_{h} \alpha_{v}^{2}$ when a single IR active mode of frequency $\omega_{h}$ is dominant, where $\alpha_{v}$ is a vibrational polarizability, and $C_{6}^{e} \approx 3 I \alpha_{e}^{2} / 4$ when a single optical absorption of energy $I$ is dominant. So the ratio is

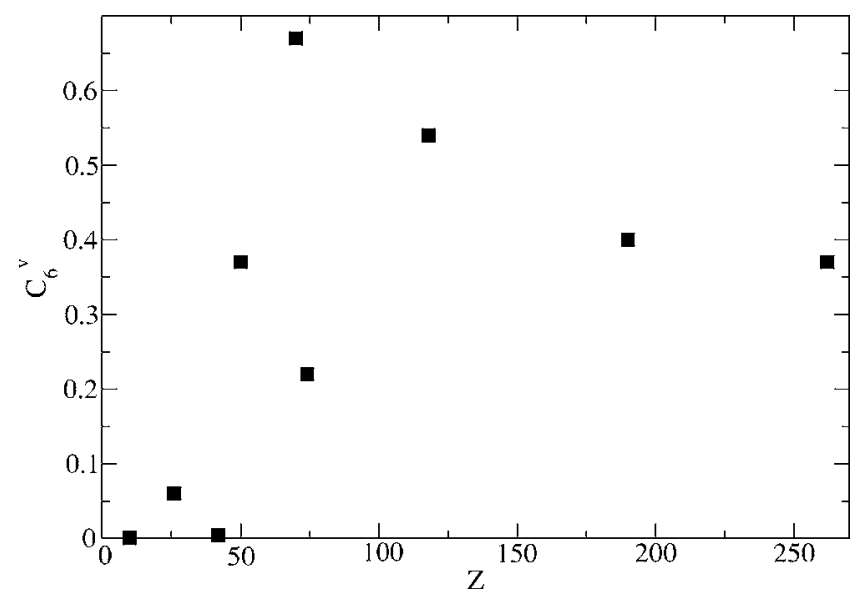

FIG. 1. Vibrational dispersion coefficient $C_{6}^{v}$ (in units of hartree $\left.\cdot a_{B}^{6}\right)$ ) vs atomic number $Z$ for the nine dimers of the same kind considered in Table II.

$$
\frac{C_{6}^{v}}{C_{6}^{e}} \approx \frac{2}{3} \frac{\hbar \omega_{h}}{I}\left(\frac{\alpha_{v}}{\alpha_{e}}\right)^{2}
$$

where $I$ is approximated by an ionization energy. In the calculation of $C_{6}^{e}[\mathrm{cal}]$, a bare atomic polarizability was considered, while in $C_{6}^{v}$ a screened vibrational polarizability was included. Therefore, to obtain a proper ratio with screening, the values of $C_{6}^{e}[\mathrm{emp}]$ and $C_{6}^{v}$ are used. The values of $C_{6}^{e}[\mathrm{emp}]$ do not seem to be correlated with the values of $C_{6}^{v}$. For example, we compare the values of $C_{6}^{e}[\mathrm{emp}]$ and $C_{6}^{v}$ for $\mathrm{C}_{6} \mathrm{H}_{6}$ to those for $\mathrm{SF}_{6}$. For the two covalent molecules investigated $\left(\mathrm{CH}_{4}\right.$ and benzene), the ratio $C_{6}^{v} / C_{6}^{e}[\mathrm{emp}]$ is in the range of $10^{-6}$. For the ionic molecules studied, such as $\mathrm{SiF}_{4}$,

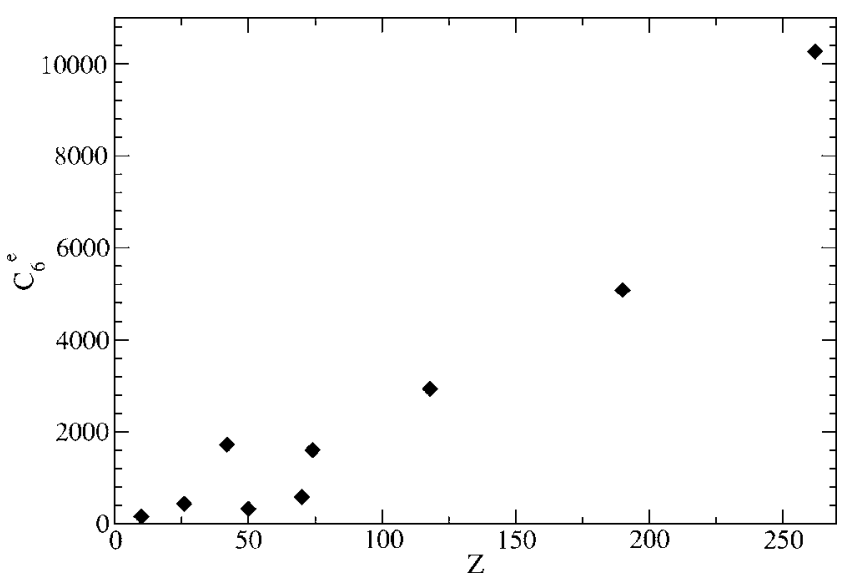

FIG. 2. Empirical electronic dispersion coefficient $C_{6}^{e}[\mathrm{emp}$ (in units of hartree $\left.\cdot a_{B}^{6}\right)$ ) vs atomic number $Z$ for the nine dimers of the same kind considered in Table II. 
TABLE III. Calculated and empirical coefficients for the electronic contribution to vdW interaction for three rare-gas dimers in units of (hartree $\cdot a_{B}^{6}$ ), using Eq. (11), Kohn-Sham orbital, and orbital energies with exchange-only (x-only) functional and exact exchange (EXX) functional, time-dependent density functional theory (TDDFT), and distributions in dipole oscillator strength (DDOS).

\begin{tabular}{lccccc}
\hline \hline & $C_{6}^{e}$ [Eq. (11)] & $C_{6}^{e}$ (x-only, Ref. 36) & $C_{6}^{e}$ (EXX, Ref.37) & $C_{6}^{e}$ (TDDFT, Ref.29) & $C_{6}^{e}$ [emp] (DDOS, Ref.25) \\
\hline $\mathrm{He}-\mathrm{He}$ & 1.565 & 1.664 & 1.72 & 1.82 & 1.458 \\
$\mathrm{Ne}-\mathrm{Ne}$ & 8.40 & 7.447 & 7.92 & 7.26 & 6.383 \\
$\mathrm{Ar}-\mathrm{Ar}$ & 108.9 & 128.5 & 131.86 & 69.9 & 64.30 \\
\hline \hline
\end{tabular}

$\mathrm{F}_{6}, \mathrm{CCl}_{4}, \mathrm{SnCl}_{4}, \mathrm{SnBr}_{4}$, and $\mathrm{SnI}_{4}$, the ratio is $10^{-5}-10^{-3}$. Since the ratio is so small, it may not be interesting to further investigate an anisotropic aspect of the vdW interaction. The ratio increases by three orders of magnitude from $\mathrm{CH}_{4}$ to $\mathrm{SiF}_{4}$ and $\mathrm{SF}_{6}$, but after that it decreases slightly as molecular weight increases according to Table II (Fig. 3). Therefore, the results shown in Table II are not sufficient to determine whether the ratio increases consistently with increasing molecular weight or if a correlation with molecular weight exists. It is, however, presumed that the ratio may not be significantly influenced by molecular weight because the crucial factor in the ratio is $\alpha_{v} / \alpha_{e}$. Among the nine molecules examined, vibrations of $\mathrm{SiF}_{4}$ and $\mathrm{SF}_{6}$ molecules contribute to the vdW interaction most. In these cases the value of $\alpha_{v}$ is half that of $\alpha_{e}$, while the frequencies of the dominant IR modes for the two dimers are about $1 \%$ of an ionization energy $I$. So the value of $C_{6}^{v}$ is approximately $0.1 \%$ of that of $C_{6}^{e}[\mathrm{emp}]$. Typical frequencies of strong IR modes for small molecules fall in the range of $200-3000 \mathrm{~cm}^{-1}(\approx 0.025-0.37 \mathrm{eV})$, while the ionization energies are typically in the range of $10 \mathrm{eV}$. Considering this, we find that the upper limit in $C_{6}^{v} / C_{6}^{e}[\mathrm{emp}]$ is approximately $1 \%$ even when $\alpha_{v} \approx \alpha_{e}$. That the purely vibrational contribution is substantially smaller than the electronic one for ionic molecules can also be understood as follows. If there exists a single dominant IR mode with a frequency of $\omega_{h}$ for a monomer, then the electronic contribution for the dimer is on the order of

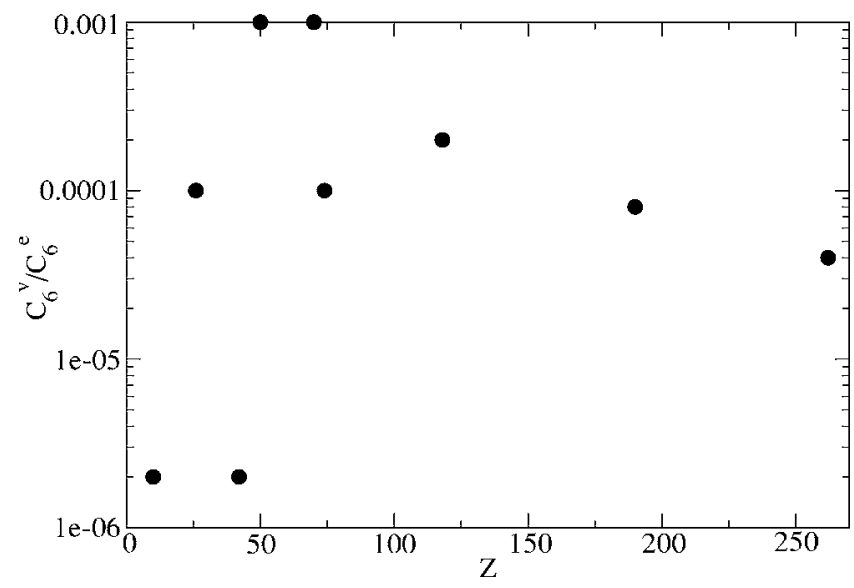

FIG. 3. Ratio $C_{6}^{v} / C_{6}^{e}[\mathrm{emp}]$ (logarithmic scale) vs $Z$ for the nine dimers of the same kind considered in Table II.

$$
\Delta U_{\mathrm{e}}^{(2)} \approx-\frac{\hbar^{4} e^{4}}{m^{2}(\hbar \omega)^{3} R^{6}} \approx-\frac{1^{4} \hbar^{4}}{1^{2} \times(10 \mathrm{eV})^{3} R^{6}}=-\frac{\hbar^{4}}{R^{6}} 10^{-3}
$$

The vibrational contribution for the dimer is

$$
\Delta U_{\mathrm{v}} \approx-\frac{1^{4} \hbar^{4}}{\left(10 \times 2 \times 10^{3}\right)^{2}(0.1 \mathrm{eV})^{3} R^{6}}=-\frac{\hbar^{4}}{R^{6}} 10^{-6} .
$$

For nonionic dimers, the electronic part does not seem to change much but the vibrational part decreases by two or three orders of magnitude because both effective charges and effective masses decrease by one or two orders of magnitude in comparison to ionic dimers.

\section{CONCLUSION}

In conclusion, we have considered an interaction between induced dipole moments caused by vibrations of atoms (or nuclei) in a molecule and compared it to an induced dipole-dipole interaction caused by fluctuations of electrons within an atom. Using second-order perturbation theory, we derived formulas for the vibrational and electronic contributions to the vdW interaction and calculated the contributions for several dimers using DFT. The calculated vibrational vdW interaction is proportional to the square of a screened vibrational polarizability. The calculated electronic part using the Kohn-Sham orbital and orbital energies is overestimated for heavy atoms due to neglect of screening of the electronic charges created by a local field. We find that the vibrational contribution is substantially smaller compared to the empirical electronic contribution (calculated using distributions in the dipole oscillator strength and the approximate London's formula) and that the upper bound could be $1 \%$ of the electronic part. Further investigation is needed to determine whether the ratio of the vibrational and electronic part could scale differently with molecular weight. According to our estimates, vibrations of atoms would play a more significant role in the vdW interaction for high-symmetry molecules with large vibrational polarizabilities and hard (high-energy) IR modes, or for low-symmetry molecules with ultrasoft IR modes and corresponding small effective masses.

\section{ACKNOWLEDGMENTS}

This work was supported in part by NSF Grant No. DMR0210717 and ONR Grant No. N00014-02-1-1046. 
*Present address: Department of Physics, Virginia Polytechnic Institute and State University, Blacksburg, VA 24061.

†Electronic address: kyungwha@vt.edu

Electronic address: pederson@dave.nrl.navy.mil

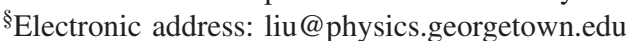

${ }^{1}$ D. C. Patton and M. R. Pederson, Phys. Rev. A 56, R2495 (1997).

${ }^{2}$ J. F. Ogilvie and F. Y. H. Wang, J. Mol. Struct. 273, 277 (1992); 291, 313 (1993).

${ }^{3}$ A. A. Quong and M. R. Pederson, Phys. Rev. B 46, 12906 (1992); M. R. Pederson and A. A. Quong, ibid. 46, 13584 (1992); A. A. Quong, M. R. Pederson, and J. L. Feldman, Solid State Commun. 87, 535 (1993).

${ }^{4}$ M. Walther, B. M. Fischer, and P. U. Jepsen, Chem. Phys. 288, 261 (2003); B. M. Fischer, M. Walther, and P. U. Jepsen, Prog. Biophys. Mol. Biol. 47, 3807 (2002).

${ }^{5}$ M. P. Jacobson, R. A. Friesner, Z. X. Xiang, and B. Honig, J. Mol. Biol. 320, 597 (2002).

${ }^{6}$ D. J. Tobias, J. E. Mertz, and C. L. Brooks, Biochemistry 30, 6054 (1991).

${ }^{7}$ M. Tarek and D. J. Tobias, Phys. Rev. Lett. 88, 138101 (2002).

${ }^{8}$ X. Y. Song and X. F. Zhao, J. Chem. Phys. 120, 2005 (2004).

${ }^{9}$ P. K. L. Drude, The Theory of Optics (Longmans, New York, 1925).

${ }^{10}$ M. Rigby, E. B. Smith, W. A. Wakeham, and G. C. Maitland, The Forces Between Molecules (Clarendon Press, Oxford, 1986).

${ }^{11}$ G. C. Maitland, M. Rigby, E. B. Smith, and W. A. Wakeham, Intermolecular Forces: Their Origin and Determination (Clarendon Press, Oxford, 1981).

${ }^{12}$ C. Kittel, Introduction to Solid State Physics, 6th ed. (John Wiley \& Sons, Inc., New York, 1986).

${ }^{13} \mathrm{H}$. Margenau and N. R. Kestner, Theory of Intermolecular Forces, 1st ed. (Pergamon Press, Hungary, 1969).

${ }^{14}$ Y. S. Kim and R. G. Gordon, J. Chem. Phys. 61, 1 (1974).

${ }^{15}$ M. Waldman and R. G. Gordon, J. Chem. Phys. 71, 1340 (1979).

${ }^{16}$ A. T. Amos and J. A. Yoffe, J. Phys. B 12, 4021 (1979).

${ }^{17}$ F. P. Billingsley II and M. Krauss, Phys. Rev. A 6, 855 (1972); W. J. Stevens and F. P. Billingsley II, ibid. 8, 2236 (1973).

${ }^{18}$ H. J. Werner and W. Meyer, Phys. Rev. A 13, 13 (1976).

${ }^{19}$ M. Krauss and D. Neumann, Chem. Phys. Lett. 52, 600 (1977).

${ }^{20}$ K. T. Tang, Phys. Rev. Lett. 23, 1271 (1969).

${ }^{21}$ K. T. Tang, J. M. Norbeck, and P. R. Certain, J. Chem. Phys. 64, 3063 (1976).

${ }^{22}$ M. E. Rosenkrantz and M. Krauss, Phys. Rev. A 32, 1402 (1985).

${ }^{23}$ G. F. Thomas, F. Mulder, and W. J. Meath, Chem. Phys. 54, 45 (1980).
${ }^{24}$ A. Kumar, G. R. G. Fairley, and W. J. Meath, J. Chem. Phys. 83, 70 (1985).

${ }^{25}$ A. Kumar and W. J. Meath, Mol. Phys. 54, 823 (1985).

${ }^{26}$ K. Rapcewicz and N. W. Ashcroft, Phys. Rev. B 44, 4032(R) (1991).

${ }^{27}$ Y. Andersson, D. C. Langreth, and B. I. Lundqvist, Phys. Rev. Lett. 76, 102 (1996); H. Rydberg, M. Dion, N. Jacobson, E. Schroder, P. Hyldgaard, S. I. Simak, D. C. Langreth, and B. I. Lundqvist, ibid. 91, 126402 (2003); M. Dion, H. Rydberg, E. Schroder, D. C. Langreth, and B. I. Lundqvist, ibid. 92, 246401 (2004).

${ }^{28}$ J. F. Dobson and B. P. Dinte, Phys. Rev. Lett. 76, 1780 (1996); J. F. Dobson and J. Wang, ibid. 82, 2123 (1999); J. F. Dobson, K. McLennan, A. Rubio, J. Wang, T. Gould, H. M. Le, and B. P. Dinte, ibid. 54, 513 (2001); J. F. Dobson, J. Wang, B. P. Dinte, K. McLennan, and H. M. Le, Int. J. Quantum Chem. 101, 579 (2005); J. F. Dobson, A. White, and A. Rubio, cond-mat/ 0507156 (unpublished).

${ }^{29}$ S. J. A. van Gisbergen, J. G. Snijders, and E. J. Baerends, J. Chem. Phys. 103, 9347 (1995).

${ }^{30}$ A. J. Misquitta, B. Jeziorski, and K. Szalewicz, Phys. Rev. Lett. 91, 033201 (2003); A. J. Misquitta and K. Szalewicz, J. Chem. Phys. 122, 214109 (2005).

${ }^{31}$ M. Gussoni, M. Rui, and G. Zerbi, J. Mol. Struct. 447, 163 (1998).

${ }^{32}$ M. R. Pederson and K. A. Jackson, Phys. Rev. B 41, 7453 (1990); K. Jackson and M. R. Pederson, ibid. 42, 3276 (1990); D. V. Porezag, Ph.D. thesis, Chemnitz Technical Institute (1997).

${ }^{33}$ J. P. Perdew, K. Burke, and M. Ernzerhof, Phys. Rev. Lett. 77, 3865 (1996).

${ }^{34}$ A. van der Merwe, Z. Phys. 196, 212 (1966); 196, 322 (1966).

${ }^{35}$ R. Eisenchitz and F. London, Z. Phys. 60, 491 (1930); F. London, Z. Phys. Chem. Abt. B 63, 245 (1930).

${ }^{36}$ M. Lein, J. F. Dobson, and E. K. U. Gross, J. Comput. Chem. 20, 12 (1999)

${ }^{37}$ A. Görling, H. H. Heinze, and M. Levy, J. Mol. Struct.: THEOCHEM 501, 271 (2000).

${ }^{38}$ D. Porezag and M. R. Pederson, Phys. Rev. B 54, 7830 (1996).

${ }^{39}$ Y. Andersson and H. Rydberg, Phys. Scr. 60, 211 (1999).

${ }^{40}$ M. R. Pederson, T. Baruah, P. B. Allen, and C. Schmidt, J. Chem. Theory Comput. 1, 590 (2005).

${ }^{41}$ A. Kumar and W. J. Meath, Mol. Phys. 75, 311 (1992).

${ }^{42}$ A. Kumar, M. Kumar, and W. J. Meath, Mol. Phys. 101, 1535 (2003). 